\title{
Enantioselective Michael addition of 2-hydroxy-1,4- naphthoquinones to nitroalkenes catalyzed by binaphthyl-derived organocatalysts
}

Saet Byeol Woo and Dae Young Kim*

\author{
Letter \\ Address: \\ Department of Chemistry, Soonchunhyang University, Asan, \\ Chungnam, 336-745, Korea \\ Email: \\ Dae Young Kim * - dyoung@sch.ac.kr \\ ${ }^{*}$ Corresponding author \\ Keywords: \\ asymmetric catalysis; Michael addition; 1,4-naphthoquinones; \\ nitroalkenes; organocatalysis
}

\author{
Beilstein J. Org. Chem. 2012, 8, 699-704. \\ doi:10.3762/bjoc. 8.78 \\ Received: 07 March 2012 \\ Accepted: 17 April 2012 \\ Published: 07 May 2012 \\ This article is part of the Thematic Series "Organocatalysis". \\ Guest Editor: B. List \\ (c) 2012 Woo and Kim; licensee Beilstein-Institut. \\ License and terms: see end of document.
}

\begin{abstract}
The highly enantioselective Michael addition of 2-hydroxy-1,4-naphthoquinones to nitroalkenes, promoted by binaphthyl-modified chiral bifunctional organocatalysts is described. This reaction afforded the chiral functionalized naphthoquinones in high yields (81-95\%) and excellent enantioselectivities (91-98\% ee) under low catalyst loading (1 mol \%).
\end{abstract}

\section{Introduction}

Quinone and naphthoquinone structures exist in a large number of natural products and biologically active molecules [1-4]. Many of these naturally occurring naphthoquinones and their synthetic analogues are important precursors for the synthesis of natural products and pharmaceuticals [5-9]. The stereoselective formation of $\mathrm{C}-\mathrm{C}$ bonds is of great importance for the synthesis of enantiomerically pure, biologically active organic compounds $[10,11]$. It is widely recognized that the Michael addition is one of the most versatile and general methods for $\mathrm{C}-\mathrm{C}$ bond formation in organic synthesis [12], and intensive research efforts have been directed toward the development of enantioselective catalytic protocols for this reaction [13-15]. The organocatalyst-mediated enantioselective conjugate addition reactions, which are both powerful and environmentally friendly, have been subjected to rigorous investigation in recent years [16-22]. The asymmetric Michael addition of various nucleophiles to nitroalkenes is of great interest, because the products obtained are versatile intermediates in organic synthesis [23-26]. Extensive studies have been devoted to the development of asymmetric conjugate additions of 1,3-dicarbonyl compounds to various Michael acceptors [27-33]. Recently, the groups of Du and Zhou reported a highly enantioselective Michael addition of 2-hydroxy-1,4-naphthoquinones to nitroalkenes catalyzed by chiral, bifunctional tertiary-amine thioureas, thiophosphorodiamides, and squaramide-based organocatalysts [34-36]. 


\section{Findings}

In the framework of our research program for the development of synthetic methods for the enantioselective construction of stereogenic carbon centers [37-42], we recently reported the enantioselective Michael addition of active methines to nitroalkenes $[43,44]$. Herein, we describe the direct enantioselective Michael addition of 2-hydroxy-1,4-naphthoquinone with nitroalkenes, catalyzed by bifunctional organocatalysts (Figure 1) that bear both central and axial chiral elements [45-47].
We initially investigated the reaction system with 2-hydroxy1,4-naphthoquinone (1) and nitrostyrene $\mathbf{2 a}$ in the presence of $10 \mathrm{~mol} \%$ of Takemoto's catalyst $\mathbf{I}$ in acetonitrile at room temperature, to determine the optimum reaction conditions for the catalytic, enantioselective Michael addition. This reaction exhibited good yield and high enantioselectivity $(89 \%$ ee, Table 1, entry 1). In order to enhance the enantioselectivity, other bifunctional organocatalysts II-VIII were evaluated in the model reaction (Table 1, entries 2-8). The quinine-derived thiourea catalyst II was less effective (Table 1, entries 1 and 2),<smiles>CN(C)[C@@H]1CCCC[C@H]1NC(=S)Nc1cc(C(F)(F)F)cc(C(F)(F)F)c1</smiles><smiles>C=CC1CN2CCC1C([C@H](NC(=S)Nc1cc(C(F)(F)F)cc(C(F)(F)F)c1)c1ccnc3ccc(OC)cc13)C2</smiles><smiles>FC(F)(F)c1cc(NC(=S)N[C@H]2CCCC[C@H]2N2Cc3ccc4ccccc4c3-c3c(ccc4ccccc34)C2)cc(C(F)(F)F)c1</smiles>

III<smiles>O=c1c(Nc2cc(C(F)(F)F)cc(C(F)(F)F)c2)c(N[C@H]2CCCC[C@H]2N2Cc3ccc4ccccc4c3-c3c(ccc4ccccc34)C2)c1=O</smiles>

VI<smiles>O=C(Nc1cc(C(F)(F)F)cc(C(F)(F)F)c1)N[C@H]1CCCC[C@H]1N1Cc2ccc3ccccc3c2-c2c(ccc3ccccc23)C1</smiles>

IV<smiles>FC(F)(F)c1cc(NC(=S)N[C@H]2CCCC[C@H]2N2Cc3ccc4ccccc4c3-c3c(ccc4ccccc34)C2)cc(C(F)(F)F)c1</smiles>

VII<smiles>O=c1c(NCc2cc(C(F)(F)F)cc(C(F)(F)F)c2)c(N[C@H]2CCCC[C@H]2N2Cc3ccc4ccccc4c3-c3c(ccc4ccccc34)C2)c1=O</smiles><smiles>O=S(=O)(N[C@H]1CCCC[C@@H]1NC(=S)N[C@H]1CCCC[C@H]1N1Cc2ccc3ccccc3c2-c2c(ccc3ccccc23)C1)c1cc(C(F)(F)F)cc(C(F)(F)F)c1</smiles>

VIII 
whereas the binaphthyl-modified, chiral, bifunctional organocatalysts III-VIII, bearing both central and axial chiral elements, effectively promoted the addition reaction in high yield, with high enantioselectivity (78-97\% ee, Table 1, entries 3-8). Catalyst III gave the desired product 3a with high enantioselectivity ( $97 \%$, Table 1 , entry 3 ), whereas the diastereomeric catalyst VII afforded product 3a in lower enantioselectivity ( $78 \%$ ee, Table 1 , entry 7$)$. These results demonstrate that the central and axial chiral elements in the chiral amine-thiourea catalyst III are matched, thus enhancing the stereochemical control, whereas in the diastereomeric catalyst VII this is not the case.

Different solvents were then tested in the presence of $10 \mathrm{~mol} \%$ of catalyst III together with 2-hydroxy-1,4-naphthoquinone (1) and nitrostyrene $\mathbf{2 a}$ in order to further improve the selectivity of the reaction. Aprotic solvents, such as acetonitrile, toluene, dichloromethane, THF, diethyl ether, were well tolerated in this conjugate addition without a significant decrease of enantio- selectivities (89-99\% ee, Table 1, entries 3 and 9-12). Remarkably, water and brine also afforded products in good yields; however, the selectivity dropped significantly (Table 1, entries 13 and 14). Among the solvents probed, the best results (92\% yield and $99 \%$ ee) were achieved when the reaction was conducted in THF (Table 1, entry 11). The present catalytic system tolerates catalyst loading down to 5, 2.5, and $1 \mathrm{~mol} \%$ without compromising the yield or enantioselectivity (Table 1 , entries 11 and 15-17).

With the optimized reaction conditions in hand, the scope of the methodology was investigated in reactions with 2-hydroxy-1,4naphthoquinone (1) and various nitroalkenes $\mathbf{2 a - 1}$ in the presence of $1 \mathrm{~mol} \%$ of catalyst III in THF at room temperature (Table 2). A range of electron-donating and electron-withdrawing substitutions on the $\beta$-aryl ring of the nitrostyrenes $\mathbf{2 b}-\mathbf{h}$ provided reaction products in high yields and excellent enantioselectivities. Heteroaryl- and naphthyl-substituted nitroalkenes $\mathbf{2} \mathbf{i}$ and $\mathbf{2} \mathbf{j}$ provided products with high selectivity

Table 1: Optimization of the reaction conditions.<smiles>CCC(C)[N+](=O)[O-]</smiles>

1

$2 a$

$3 a$

\begin{tabular}{|c|c|c|c|c|c|}
\hline entry & cat. & solvent & time (h) & yield $(\%)^{a}$ & ee $(\%)^{b}$ \\
\hline 1 & I & $\mathrm{CH}_{3} \mathrm{CN}$ & 2 & 84 & 89 \\
\hline 2 & II & $\mathrm{CH}_{3} \mathrm{CN}$ & 2 & 87 & 77 \\
\hline 3 & III & $\mathrm{CH}_{3} \mathrm{CN}$ & 2 & 96 & 97 \\
\hline 4 & IV & $\mathrm{CH}_{3} \mathrm{CN}$ & 2 & 95 & 87 \\
\hline 5 & $\mathbf{V}$ & $\mathrm{CH}_{3} \mathrm{CN}$ & 2 & 93 & 81 \\
\hline 6 & VI & $\mathrm{CH}_{3} \mathrm{CN}$ & 2 & 90 & 93 \\
\hline 7 & VII & $\mathrm{CH}_{3} \mathrm{CN}$ & 2 & 85 & 78 \\
\hline 8 & VIII & $\mathrm{CH}_{3} \mathrm{CN}$ & 2 & 88 & 93 \\
\hline 9 & III & toluene & 4 & 75 & 95 \\
\hline 10 & III & DCM & 4 & 93 & 89 \\
\hline 11 & III & THF & 2 & 92 & 99 \\
\hline 12 & III & $\mathrm{Et}_{2} \mathrm{O}$ & 3 & 81 & 91 \\
\hline 13 & III & $\mathrm{H}_{2} \mathrm{O}$ & 17 & 89 & 19 \\
\hline 14 & III & brine & 17 & 86 & 37 \\
\hline $15^{c}$ & III & $\mathrm{THF}$ & 2 & 90 & 98 \\
\hline $16^{d}$ & III & THF & 2 & 90 & 99 \\
\hline $17^{e}$ & III & THF & 2 & 89 & 99 \\
\hline
\end{tabular}

alsolated yield.

bEnantiopurity was determined by HPLC analysis using chiralcel OJ-H column.

${ }^{\mathrm{C}}$ Reaction was carried out in the presence of $5 \mathrm{~mol} \%$ catalyst.

${ }^{\mathrm{d}}$ Reaction was carried out in the presence of $2.5 \mathrm{~mol} \%$ catalyst.

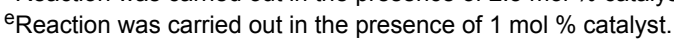


Table 2: Catalytic asymmetric Michael addition of 2-hydroxy-1,4-naphthoquinone 1 to nitroalkenes 2.

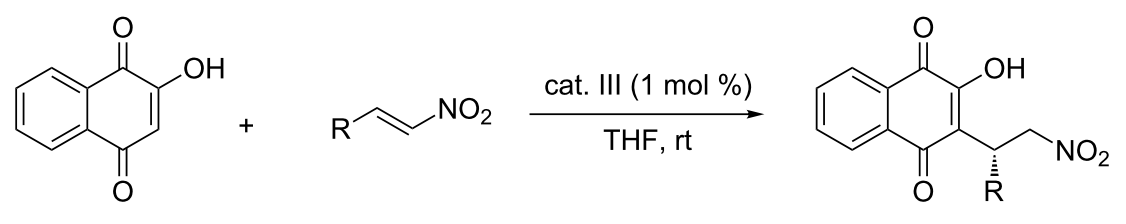

1

2

3

\begin{tabular}{|c|c|c|c|c|}
\hline entry & 2, R & time $(\mathrm{h})$ & yield (\%) & ee $(\%)^{b}$ \\
\hline 1 & $\mathbf{2 a}, \mathrm{Ph}$ & 2 & $3 a, 89$ & 99 \\
\hline 2 & 2b, p- $\mathrm{MeC}_{6} \mathrm{H}_{4}$ & 2 & $3 b, 93$ & 95 \\
\hline 3 & 2c, $p-\mathrm{MeOC}_{6} \mathrm{H}_{4}$ & 4 & $3 c, 81$ & 99 \\
\hline 4 & 2d, $p-\mathrm{FC}_{6} \mathrm{H}_{4}$ & 3 & 3d, 95 & 95 \\
\hline 5 & $2 \mathrm{e}, p-\mathrm{ClC}_{6} \mathrm{H}_{4}$ & 3 & $3 e, 90$ & 91 \\
\hline 6 & 2f, $p-\mathrm{BrC}_{6} \mathrm{H}_{4}$ & 3 & $3 f, 95$ & 95 \\
\hline 7 & $2 \mathrm{~g}, \mathrm{o}-\mathrm{FC}_{6} \mathrm{H}_{4}$ & 4 & $3 g, 95$ & 95 \\
\hline 8 & 2h, o- $\mathrm{BrC}_{6} \mathrm{H}_{4}$ & 4 & $3 h, 95$ & 95 \\
\hline 9 & 2i, 2-thienyl & 5 & $3 \mathbf{i}, 93$ & 93 \\
\hline 10 & 2j, 2-naphthyl & 5 & $3 \mathbf{j}, 93$ & 99 \\
\hline 11 & 2k, isobutyl & 5 & $3 \mathbf{k}, 90$ & 97 \\
\hline
\end{tabular}

alsolated yield.

${ }^{b}$ Enantiopurity was determined by HPLC analysis using chiralcel OJ-H (3a-j) and chiralpak AD-H (for $\mathbf{3 k}$ ) columns.

(93-99\% ee, Table 2, entries 9 and 10). The $\beta$-alkyl-substituted nitroalkene, 4-methyl-1-nitropent-1-ene (2k), was also an acceptable starting material and provided the corresponding Michael adducts in high yield and excellent enantioeselectivity (97\% ee, Table 2, entry 11$)$.

In conclusion, we have developed a highly efficient catalytic, enantioselective Michael addition of 2-hydroxy-1,4-naphthoquinone to nitroalkenes using a binaphthyl-derived tertiary amine-thiourea organocatalyst. The various types of nitroalkylated naphthoquinone derivatives were obtained in good to high yields with excellent enantioselectivities (91-99\% ee) for all the substrates examined in this work. We believe that this method should provide a practical entry for the preparation of chiral nitroalkylated naphthoquinone derivatives. Further details and application of this asymmetric Michael addition of 2-hydroxy1,4-naphthoquinone nucleophiles will be presented in due course.

\section{Experimental}

General procedure for the Michael addition of 2-hydroxy1,4-naphthoquinone (1) with nitroalkenes 2: A mixture of 2-hydroxy-1,4-naphthoquinones $(\mathbf{1}, 34.8 \mathrm{mg}, 0.2 \mathrm{mmol})$ and catalyst III (1.3 mg, $0.002 \mathrm{mmol})$ in THF $(0.4 \mathrm{~mL})$ was stirred at room temperature for $5 \mathrm{~min}$. A solution of nitroalkene 2 $(0.2 \mathrm{mmol})$ was added. The reaction mixture was stirred for
2-5 $\mathrm{h}$ at room temperature. After completion of the reaction, the resulting solution was concentrated in vacuo and the obtained residue was purified by flash chromatography (EtOAc-hexane) to afford the corresponding Michael adducts $\mathbf{3}$. Products $\mathbf{3}$ are known compounds, and their data were identical to those reported in the literature [34-36].

\section{Supporting Information}

\section{Supporting Information File 1}

Characterization data of products 3 .

[http://www.beilstein-journals.org/bjoc/content/ supplementary/1860-5397-8-78-S1.pdf]

\section{Acknowledgements}

This work was supported in part by the Soonchunhyang University Research Fund.

\section{References}

1. de Andrade-Neto, V. F.; Goulart, M. O. F.; da Silva Filho, J. F.; da Silva, M. J.; Pinto, M. C. F. R.; Pinto, A. V.; Zalis, M. G.; Carvalho, L. H.; Krettli, A. U. Bioorg. Med. Chem. Lett. 2004, 14, 1145-1149. doi:10.1016/j.bmcl.2003.12.069

2. Tandon, V. K.; Yadav, D. B.; Singh, R. V.; Chaturvedi, A. K.; Shukla, P. K. Bioorg. Med. Chem. Lett. 2005, 15, 5324-5328. doi:10.1016/j.bmcl.2005.08.032 
3. Glänzel, M.; Bültmann, R.; Starke, K.; Frahm, A. W. Eur. J. Med. Chem. 2005, 40, 1262-1276. doi:10.1016/j.ejmech.2005.07.007

4. Gomez-Monterrey, I.; Santelli, G.; Campiglia, P.; Califano, D.; Falasconi, F.; Pisano, C.; Vesci, L.; Lama, T.; Grieco, P.; Novellino, E. J. Med. Chem. 2005, 48, 1152-1157. doi:10.1021/jm0408565

5. Gomez-Monterrey, I.; Campiglia, P.; Carotenuto, A.; Califano, D.; Pisano, C.; Vesci, L.; Lama, T.; Bertamino, A.; Sala, M.; Mazzella di Bosco, A.; Grieco, P.; Novellino, E. J. Med. Chem. 2007, 50, 1787-1798. doi:10.1021/jm0612158

6. Castellano, S.; Bertamino, A.; Gomez-Monterrey, I.; Santoriello, M.; Grieco, P.; Campiglia, P.; Sbardella, G.; Novellino, E. Tetrahedron Lett. 2008, 49, 583-585. doi:10.1016/j.tetlet.2007.11.148

7. Hsin, L.-W.; Wang, H.-P.; Kao, P.-H.; Lee, O.; Chen, W.-R.; Chen, H.-W.; Guh, J.-H.; Chan, Y.-L.; His, C.-P.; Yang, M.-S.; Li, T.-K.; Lee, C.-H. Bioorg. Med. Chem. 2008, 16, 1006-1014. doi:10.1016/j.bmc.2007.10.012

8. Wei, P.; Zhang, X.; Tu, S.; Yan, S.; Ying, H.; Ouyang, P. Bioorg. Med. Chem. Lett. 2009, 19, 828-830. doi:10.1016/j.bmcl.2008.12.006

9. Zhang, G.; Wang, Y.; Zhang, W.; Xu, X.; Zhong, A.; Xu, D. Eur. J. Org. Chem. 2011, 2142-2147. doi:10.1002/ejoc.201001570

10. Corey, E. J.; Guzman-Perez, A. Angew. Chem., Int. Ed. 1998, 37, 388-401. doi:10.1002/(SICI)1521-3773(19980302)37:4<388::AID-ANIE388>3.0. $\mathrm{CO} ; 2-\mathrm{V}$

11. Christoffers, J.; Mann, A. Angew. Chem., Int. Ed. 2001, 40, 4591-4597. doi:10.1002/1521-3773(20011217)40:24<4591::AID-ANIE4591>3.0.CO ;2-V

12. Leonard, J. Contemp. Org. Synth. 1994, 1, 387-415. doi:10.1039/CO9940100387

13. Krause, N.; Hoffmann-Röder, A. Synthesis 2001, 171-196. doi:10.1055/s-2001-10803

14. Berner, O. M.; Tedeschi, L.; Enders, D. Eur. J. Org. Chem. 2002, 1877-1894. doi:10.1002/1099-0690(200206)2002:12<1877::AID-EJOC1877>3.0.C O;2-U

15. Christoffers, J.; Baro, A. Angew. Chem., Int. Ed. 2003, 42, 1688-1690. doi:10.1002/anie.200201614

16. Connon, S. J. Angew. Chem., Int. Ed. 2006, 45, 3909-3912. doi:10.1002/anie.200600529

17. Tylor, M. S.; Jacobson, E. N. Angew. Chem., Int. Ed. 2006, 45, 1520-1543. doi:10.1002/anie.200503132

18. Doyle, A. G.; Jacobsen, E. N. Chem. Rev. 2007, 107, 5713-5743. doi:10.1021/cr068373r

19. Yu, X.; Wang, W. Chem.-Asian J. 2008, 3, 516-532. doi:10.1002/asia.200700415

20. Connon, S. J. Synlett 2009, 354-376. doi:10.1055/s-0028-1087557

21. Tsogoeva, S. B. Eur. J. Org. Chem. 2007, 1701-1716. doi:10.1002/ejoc.200600653

22. Almaşi, D.; Alonso, D. A.; Nájera, D. Tetrahedron: Asymmetry 2007, 18, 299-365. doi:10.1016/j.tetasy.2007.01.023

23. Ono, N. The Nitro Group in Organic Synthesis; Wiley-VCH: New York, 2001.

24. Calderari, G.; Seebach, D. Helv. Chim. Acta 1985, 68, 1592-1604. doi:10.1002/hlca.19850680611

25. Ballini, R.; Petrini, M. Tetrahedron 2004, 60, 1017-1047. doi:10.1016/j.tet.2003.11.016

26. Czekelius, C.; Carreira, E. M. Angew. Chem., Int. Ed. 2005, 44, 612-615. doi:10.1002/anie.200461879
27. Hamashima, Y.; Hotta, D.; Sodeoka, M. J. Am. Chem. Soc. 2002, 124, 11240-11241. doi:10.1021/ja027075i

28. Wu, F.; Li, H.; Hong, R.; Deng, L. Angew. Chem., Int. Ed. 2006, 45, 947-950. doi:10.1002/anie.200502658

29. Kang, Y. K.; Kim, D. Y. Tetrahedron Lett. 2006, 47, 4565-4568. doi:10.1016/j.tetlet.2006.05.003

30. Bartoli, G.; Bosco, M.; Carlone, A.; Cavalli, A.; Locatelli, M.; Mazzanti, A.; Ricci, P.; Sambri, L.; Melchiorre, P. Angew. Chem., Int. Ed. 2006, 45, 4966-4970. doi:10.1002/anie.200600370

31. Rigby, C. L.; Dixon, D. J. Chem. Commun. 2008, 3798-3800. doi:10.1039/B805233F

32. Jung, S. H.; Kim, D. Y. Tetrahedron Lett. 2008, 49, 5527-5530. doi:10.1016/j.tetlet.2008.07.041

33. Capuzzi, M.; Perdicchia, D.; Jørgensen, K. A. Chem.-Eur. J. 2008, 14, 128-135. doi:10.1002/chem.200701317

34. Zhou, W.-M.; Liu, H.; Du, D.-M. Org. Lett. 2008, 10, 2817-2820. doi:10.1021/ol800945e

35. Wu, R.; Chang, X.; Lu, A.; Wang, Y.; Wu, G.; Song, H.; Zhou, Z.; Tang, C. Chem. Commun. 2011, 47, 5034-5036. doi:10.1039/c1cc10797f

36. Yang, W.; Du, D.-M. Adv. Synth. Catal. 2011, 353, 1241-1246. doi:10.1002/adsc.201000981

37. Kang, Y. K.; Kwon, B. K.; Mang, J. Y.; Kim, D. Y. Tetrahedron Lett. 2011, 52, 3247-3249. doi:10.1016/j.tetlet.2011.04.084

38. Kang, Y. K.; Suh, K. H.; Kim, D. Y. Synlett 2011, 1125-1128. doi:10.1055/s-0030-1259932

39. Kang, Y. K.; Kim, D. Y. Tetrahedron Lett. 2011, 52, 2356-2358. doi:10.1016/j.tetlet.2011.02.087

40. Kang, S. H.; Kim, D. Y. Adv. Synth. Catal. 2010, 352, 2783-2786. doi:10.1002/adsc.201000515

41. Moon, H. W.; Kim, D. Y. Tetrahedron Lett. 2010, 51, 2906-2908. doi:10.1016/j.tetlet.2010.03.105

42. Kang, Y. K.; Kim, S. M.; Kim, D. Y. J. Am. Chem. Soc. 2010, 132, 11847-11849. doi:10.1021/ja103786c

43. Kwon, B. K.; Kim, S. M.; Kim, D. Y. J. Fluorine Chem. 2009, 130, 759-761. doi:10.1016/j.jfluchem.2009.06.002

44. Oh, Y.; Kim, S. M.; Kim, D. Y. Tetrahedron Lett. 2009, 50, 4674-4676. doi:10.1016/j.tetlet.2009.06.003

45. Lee, H. J.; Kang, S. H.; Kim, D. Y. Synlett 2011, 1559-1562. doi:10.1055/s-0030-1260770

46. Yoon, S. J.; Kang, Y. K.; Kim, D. Y. Synlett 2011, 420-424. doi:10.1055/s-0030-1259319

47. Kim, S. M.; Lee, J. H.; Kim, D. Y. Synlett 2008, 2659-2662. doi:10.1055/s-0028-1083510 


\section{License and Terms}

This is an Open Access article under the terms of the Creative Commons Attribution License

(http://creativecommons.org/licenses/by/2.0), which permits unrestricted use, distribution, and reproduction in any medium, provided the original work is properly cited.

The license is subject to the Beilstein Journal of Organic Chemistry terms and conditions:

(http://www.beilstein-journals.org/bjoc)

The definitive version of this article is the electronic one which can be found at:

doi: $10.3762 /$ bjoc. 8.78 